\title{
SYSTEM LEVEL SIMULATION FOR TWO TIER MACRO-FEMTO CELLULAR NETWORKS
}

\author{
${ }^{1}$ Shiqi Xing, ${ }^{2}$ Pantha Ghosal, ${ }^{3}$ Shouman Barua, ${ }^{4}$ Ramprasad Subramanian and \\ ${ }^{5}$ Kumbesan Sandrasegaran \\ Centre for Real-time Information Networks \\ School of Computing and Communications, Faculty of Engineering and Information \\ Technology, University of Technology Sydney, Sydney, Australia.
}

\begin{abstract}
LTE is an emerging wireless communication technology to provide high-speed data service for the mobile phones and data terminals. To improve indoor coverage and capacity Femtocells are included in 3GPP since Release 8. There is no common simulation platform is available for performance justification of LTEFemtocells. LTE-Sim is an object-oriented open source simulator which incorporates a complete protocol stack can be used for simulating two-tier macro-femto scenarios. To the best of our knowledge no paper provides the guideline to perform system level simulation of Femtocell networks. Here, in this paper Femtocells performance is evaluated in multi-Macrocells and multi-Femtocells environment with interference from Microcells and Macrocell users along with the scripting.
\end{abstract}

\section{KEYWORDS}

Channel quality indicator (CQI), Femto Access Point (FAP), Macro eNodeB (MeNB), Macrocell User Equepment (MUE), Moblity Management Entity(MME), Signal to Interference Plus Noise Ratio(SINR), Physical Layer(PHY)

\section{INTRODUCTION}

Long Term Evaluation (LTE) is the next generation communication technology. LTE, which supports high data transmission rate has upgraded the major network infrastructure by legacy networks (GSM, GPRS, UMTS/HSPA) with the combination of circuit-switched and packetswitched technology. With the higher frequency bands allocated to LTE, sometimes indoor coverage becomes poor since penetration and path loss is higher for higher frequency bands. Addressing the challenge of indoor coverage, and to provide high data transmission rate to users, small, portable, short ranged $(10-35 \mathrm{~m})$, low powered (10-100mW) Femto access [1] points were integrated in 3GPP. The benefits of Femtocells include high coverage, high mobile data capacity, improve battery life of mobile devices in the next generation wireless communication system. Femtocells is operating at the frequency spectrum owned by the service operator and enable fixed mobile convergence (FMC) [2] service by connecting to the core network via broadband communication links such as Digital Subscriber Line (DSL). The portability and low cost makes femtocells deployed by the owner in an unplanned manner; also the dense installation of femtocells increases the complexity in mobility management and increases interference. For that 
International Journal of Wireless \& Mobile Networks (IJWMN) Vol. 6, No. 6, December 2014

purpose research on performance evaluation on accurate simulation framework is of utmost importance. The current open-source simulation tools are only able to simulate parts of the LTE system [3] but we are not aware of any simulator that allows femtocell architecture the media access control (MAC) and physical layer (PHY) models with handover strategies and propagation loss models for indoor environments. To bridge this gap, this paper describes our efforts to build multi-cell and multi-user environment in femtocellular module in the existing open source LTESim framework [4] encompassing scenarios with both macro and femto cells

The rest of the paper is organized as, Section II. LTE Network Architecture with Femtocells. Section III. Modelling and Coding in LTE-Sim. Section IV explains the simulation outcomes of Femtocells module in LTE-Sim. Section V concludes the paper.

\section{LTE FEMTOCELL: NeTWORK ARCHITECTURE}

\subsection{LTE and Scheduling Frame}

The LTE network architecture is divided into two parts: the air interfaces E-UTRAN, and the packet switched core network (EPC) [5]. On the network side, the evolved NodeB (eNB) is the only component of E-UTRAN, the main functionalities are 1) sending and receiving radio transmission to all mobiles and data terminals in downlink and uplink respectively and 2) controlling singling messages (such as Handover message) On the physical layer, LTE radio interfaces supports both of Frequency Division Duplexing (FDD) and Time Division Duplexing (TDD). Channel is based on Orthogonal Frequency Division Multiple Access (OFDMA). The use of OFDMA removes the Inter Symbol Interferences since the transmitter uses a guard period (cyclic prefix) longer than the channel response [6]. Radio resources are allocated in a time/frequency domain. In the time domain, every Transmission Time Interval (TTI) has been distributed into $1 \mathrm{~ms}$. Each TTI is composed of two consecutive time slots of $0.5 \mathrm{~ms}$ and seven OFDM symbols with short cyclic prefix. On the other side, in the frequency domain, the bandwidth is divided into $180 \mathrm{kHz}$ sub-channels, corresponding to 12 consecutive and equally spaced sub-carriers.

\subsection{Femtocell and Its challenges}

Femtocell was introduced by 3GPP in Release 8 which has the functionalities of boosting the indoor coverage and capacity. Femtocell may also improve battery life of mobile devices. Fig. 1 shows the basic femtocell network architecture of macro-femto two-layer systems. According to [3], femtocell access points are connected to the operator network through a DSL line at end user like a common Wi-Fi access point. 
International Journal of Wireless \& Mobile Networks (IJWMN) Vol. 6, No. 6, December 2014

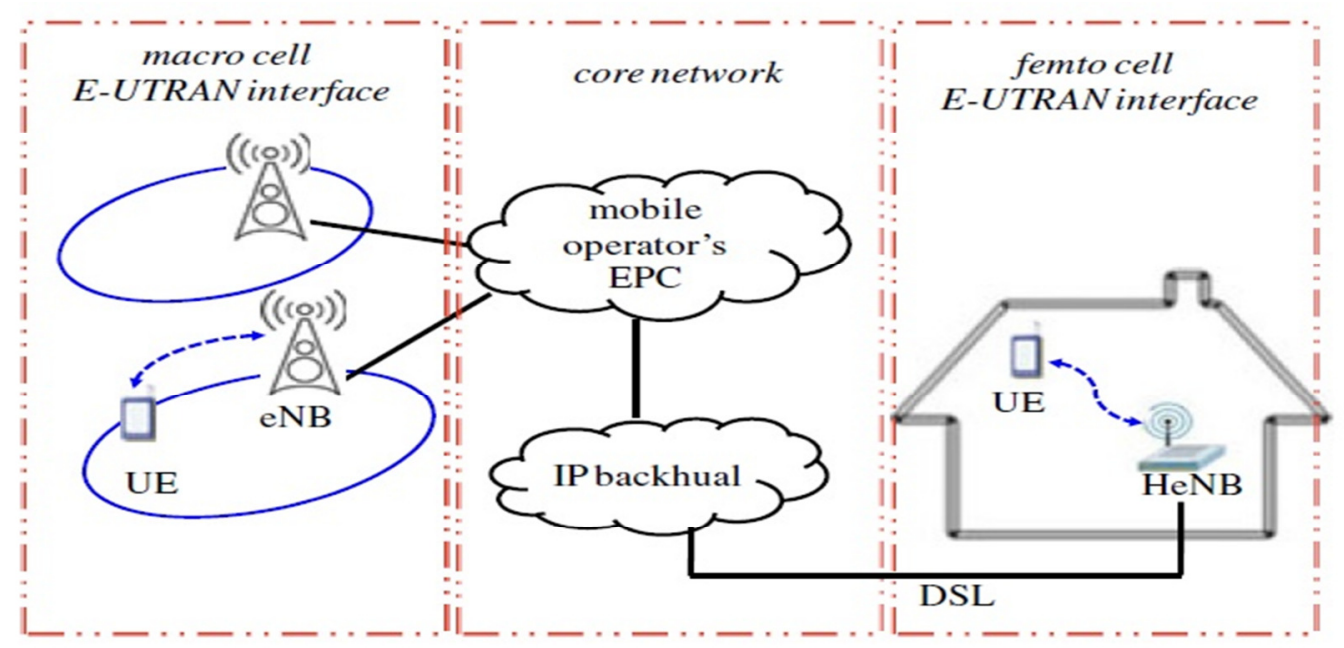

Fig.1. UEs inside building served by Femtocell

LTE system is operating on flat IP architecture which connects all the eNBs through X2 interfaces and Radio Resource Management (RRM) procedure is done by eNB. On the other hand, there is no such X2 interface between eNB and FAP. As a result, Interference coordination [7], RRM and HO management [8] are becoming complex with the unplanned and vast deployment of LTE femtocells. Benefits of femtocells are coming with the increasing the complexity in spectrum management. Femtocells are designed to operate in one of the three different access modes, i.e., closed access mode, open access mode or hybrid access mode [9]. In closed access mode, the UEs belong to the Closed Subscriber Group are allowed to get access to the femtocell. However, the unauthorized users might affect by the interference from the femtocells in closed access mode. In the case of open access mode, any UE could get access to the femtocells and benefit from its services. On the other hand, depending on resource usage, congestion and security consideration, open access is not a suitable choice. For hybrid access mode, a limited number of unknown Macro UEs are allowed to access the femtocell by owners, but the owners will suffer the risk of a security breach [10].

\subsection{Femtocell Hand Over Modeling}

According to [5], Fig. 2 illustrates three femtocell handover scenarios: Inbound Handover, i.e., the handover that takes place when user moves from the eNB to HeNB. Outbound scenario denotes the handover from the HeNB to eNB. Inter FAP, i.e., UE handover from one femtocell to other femtocells. Fig. 3 shows the Femtocell UE hand over to another femtocell. 
International Journal of Wireless \& Mobile Networks (IJWMN) Vol. 6, No. 6, December 2014

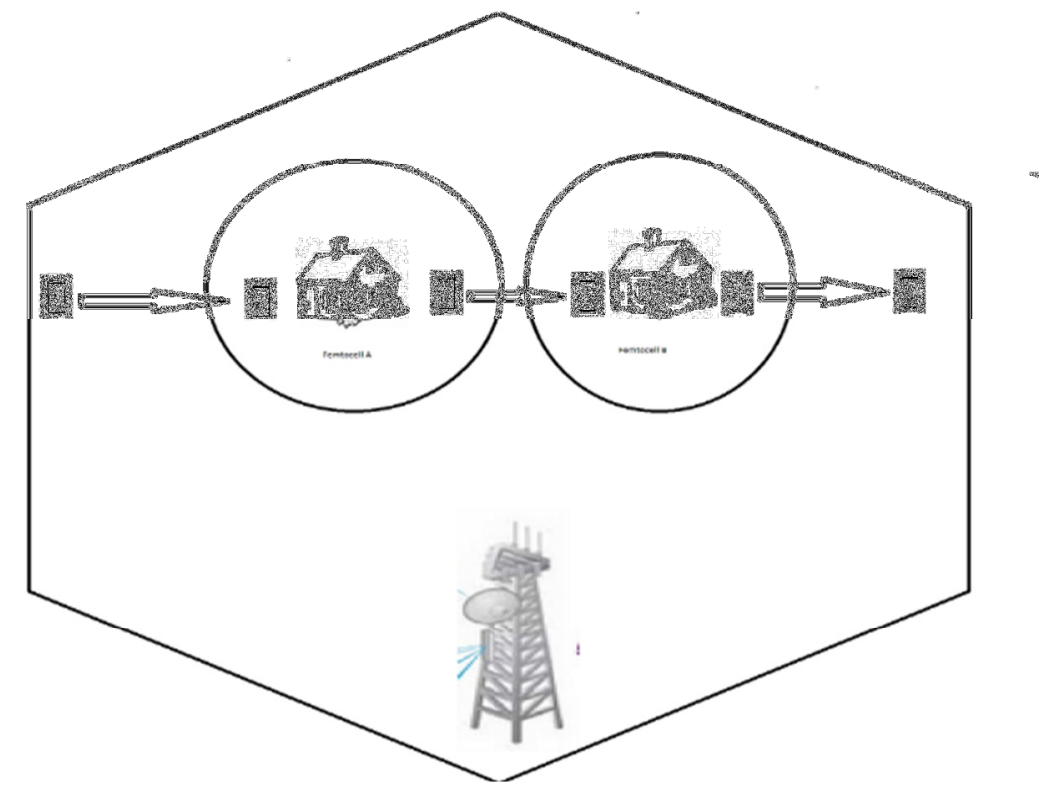

Fig.2: Handover modeling in presence of Femtocells [5]

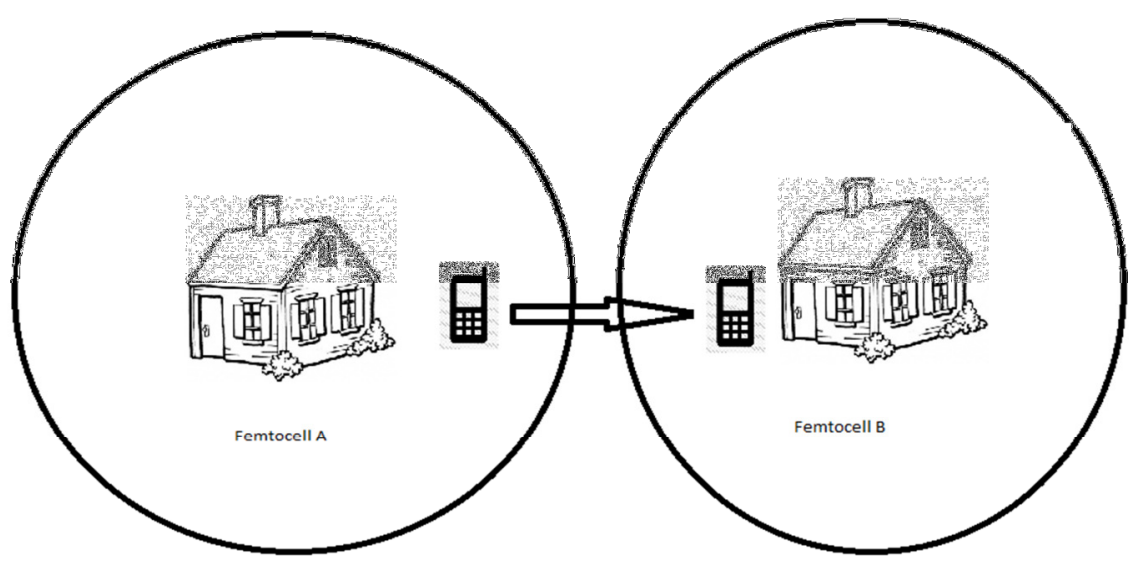

Fig.3: Handover between Femtocells. [5]

\subsection{Frequency reuse modeling}

LTE supports Fractional Frequency Reuse. The main idea of FFR is to divide allocated spectrum into several sub-bands and allocate these sub-bands in different macro cell coverage area or subarea [6]. Since, the resources are not overlapped in different sub-areas the cross-tier interference between FAP and eNB can be mitigated. In [11] authors proposed "soft frequency reuse" scheme where frequency spectrum is divided in centre and edge zones by adjusting transmitting power for effective reuse. 

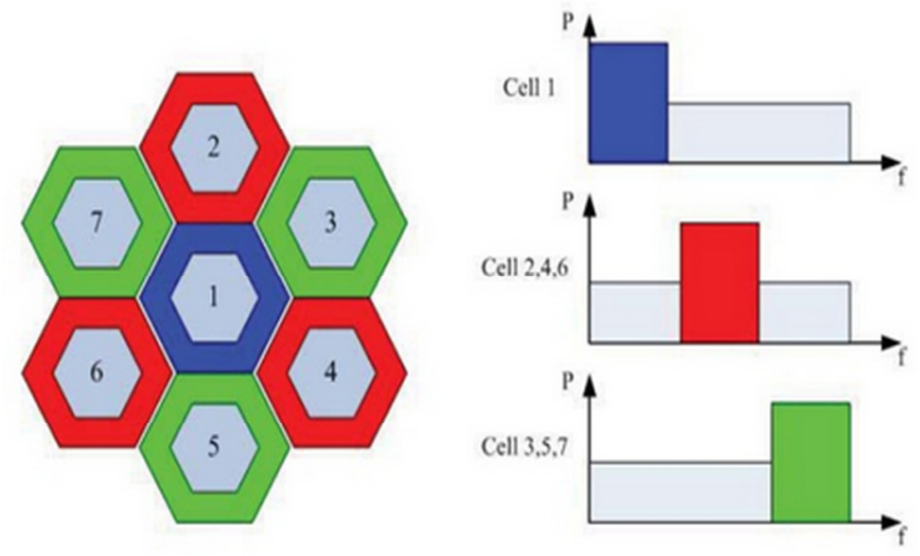

Fig.4: Frequency Planning and Power Allocation for SFR Scheme [11]

In Soft Frequency Reuse, the sub bands are orthogonal to each other and users at cell edge are allocated a cell-edge band as illustrated in Fig. 4 with cluster of 7 cells. Users in the cell edge band have higher power whereas Cell Centre Users of neighbouring cells also have access to the selected cell edge bands but with lower power. Therefore, high spectral efficiency could be achieved with user throughput. Though, intracell interference is reduced because of orthogonally between bands but UEs experience higher ICI because the Orthogonality between cell edge band of one cell and cell-interior bands of its neighbouring cells is not guaranteed. Strict FFR as shown in Fig.4 is used mostly in multi cell networks and is actually a modification of traditional Frequency reuse with a cell-edge reuse factor. In Fig.5, for the three cells illustrated, the cell centre area is allocated the common sub-band while at the cell-edge remaining bandwidth is portioned with a reuse factor 3. Here, cell centre users don't share spectrum with exterior user and reduce cross tier interference

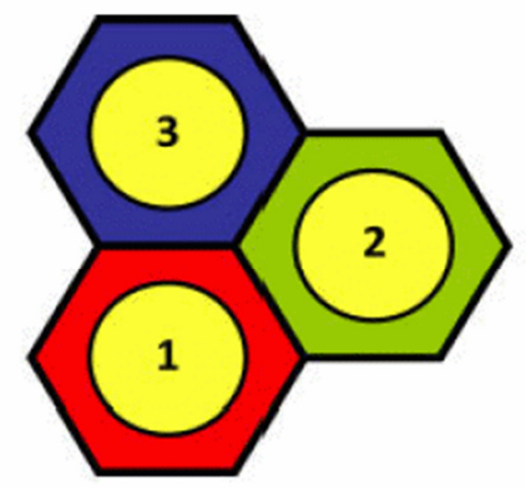

Cell 1

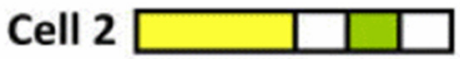

Cell 3

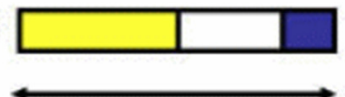

Total Bandwidth 
Fig.5: Strict FFR [14]

In reuse factor 3, the whole frequency band can be divided into three equal, orthogonal sub-bands, assigned to sectors so that adjacent sectors always use different frequencies as shown if Fig. 6. FFR3 leads to low improved ICI but large capacity is lost because only one third of the resources are used in each cell.
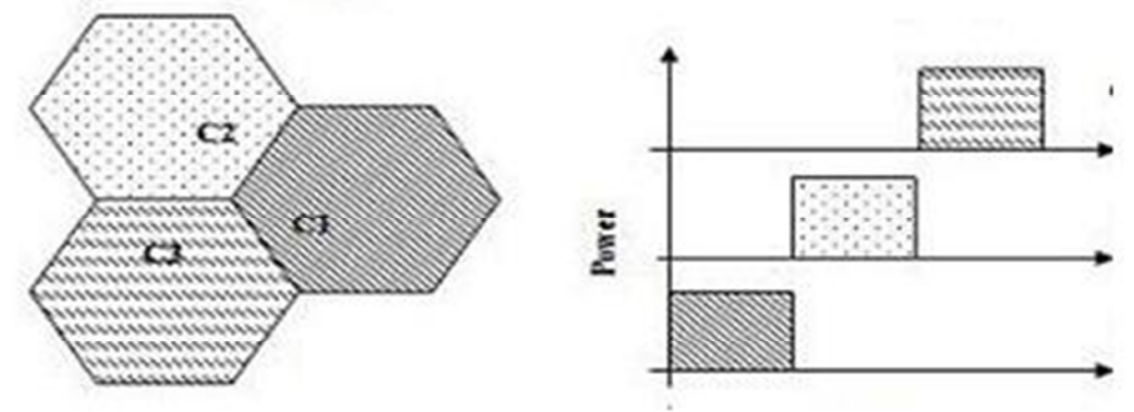

Fig.6: Fractional Frequency Reuse-3

\section{LTE-Sim: FEMTOCELL Simulation}

\subsection{LTE-Sim Background}

LTE-Sim is a LTE network system simulator which written in well-known object-oriented C++ platform [3]. It has the functionalities of simulating several aspects of LTE network, such as EUTRAN and EPC, downlink and uplink transmissions, single and multi-cell environments, QoS management, multiuser environment, user mobility, handover procedures, and frequency reuse techniques. In addition, the entire LTE protocol stack is framed from the application to the PHY layer, such as the Radio Resource Control (RRC), Radio Link Control (RLC) and MAC entities. Furthermore, LTE-Sim supports scheduling strategies (such as Proportional Fair, Log and Exp/PF rules). Moreover, LTE-Sim is operating on shell script. After building up the scenario in C++, shell script program works as an interface to start the simulation and analyse the simulation results.

\subsection{Creating Femtocell Building in simulator scenario}

Creating simulation scenario with femtocell includes constructing the macro cells, femtocell building, femtocells, macro cell UEs and femtocell UEs in LTE-Sim. FAPs and eNBs are identified by unique IDs and their positions are defined by Cartesian Coordinate program function [3]. Position tracking, information related to all registered UEs, like the UE identifier, CQI feedbacks, uplink channel quality, and uplink scheduling can be simulated by defining the functionalities debug in the global-config document. In LTE-Sim, a building is composed of a number of apartments and in this simulation each one defines the area of a given femtocell. According to the definition in [8], two different types of building are developed and used in LTESim: (a) Dual Stripe blocks and (b) $5 \times 5$ apartment grid. The former consists two building rows composed of 10 apartments each. The later is a building of 25 apartments located over a $5 \times 5$ grid. This apartment grid can also be modified according to the traffic environment. In this paper, a modified apartment grid $(3 \times 3)$ as Fig. 7 is considered for simulation. Each apartment has a squared form and an area of $100 \mathrm{~m}^{2}$. This means, for instance, a building with $3 \times 3$ apartment 
International Journal of Wireless \& Mobile Networks (IJWMN) Vol. 6, No. 6, December 2014

grid can contain up to 9 femtocells. Grid of apartments inside of the building could be defined by configuring the grid settings (i.e. building types) in the header file (SingleCellWithFemto.h). Building walls are formed in NetworkManager.cpp where, function

NetworkManager::CreateBuildingForFemtocells() gets building ID defined in SingleCellWithFemto.h and creates the building according to the building type as presented in Fig. 7.

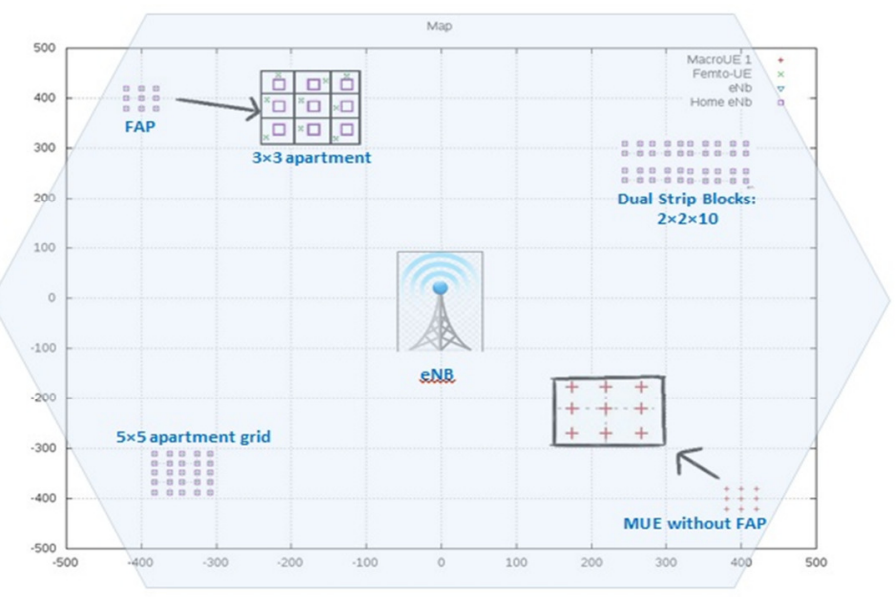

Fig. 7: Femtocell design

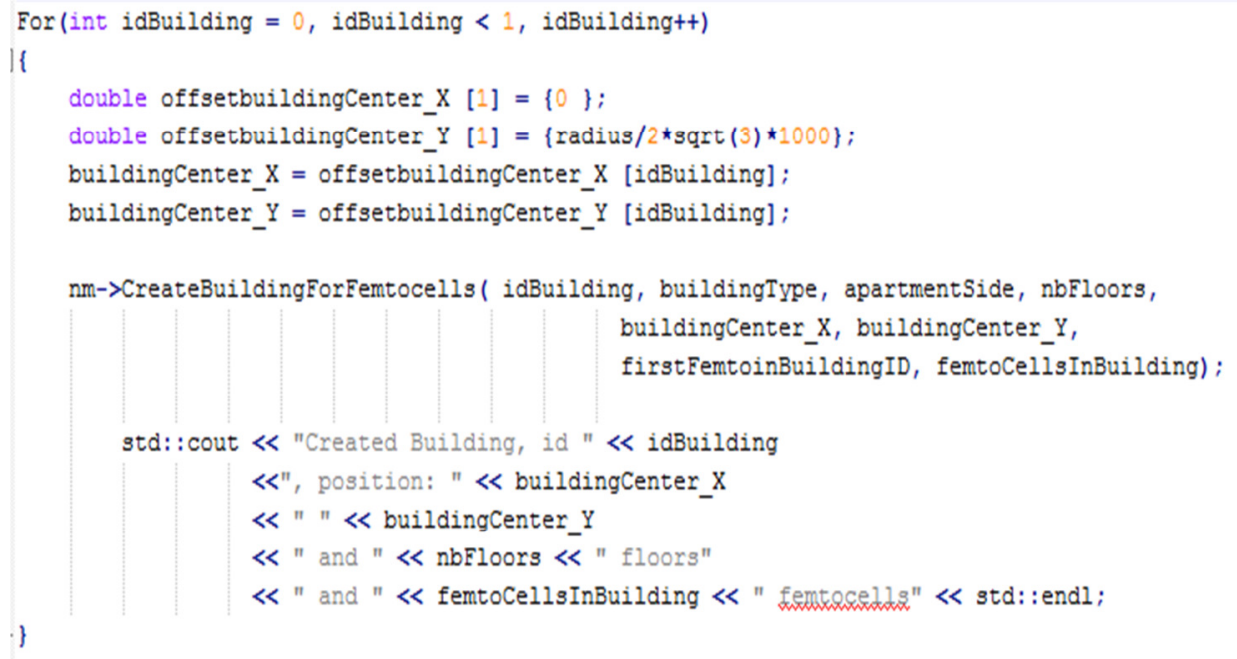

Code Block.1: Positioning of Building containing Femtocells

Code Block.1 shows the positioning of a building containing femtocells (here, the building location is set at $\mathrm{X}=0, \mathrm{Y}=866$ coordinates) where, the coverage area of eNB is considered $1000 \mathrm{~m}$. 


\subsection{Create UE in simulator}

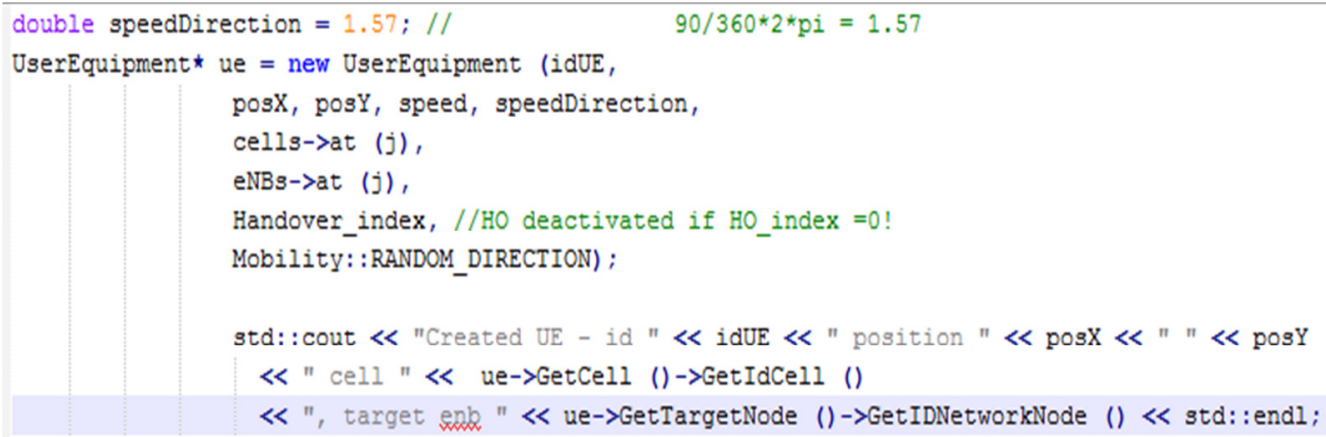

Code Block.2: Mobility of UE in different HO scenarios

In Code Block 2, it is shown how to create an user at coordinte $(0,0)$ with mobility to perform different HO in different scenarios. In our simulation we considered Random Direction[12] as our mobility model. Here, the UE is moving North, as shown in Fig.8 will pass through femtocell building and handover form Macrocell 1 -> Femtocell 1 -> Femtocell 2 -> Femtocell No.3 -> Macrocell 2.

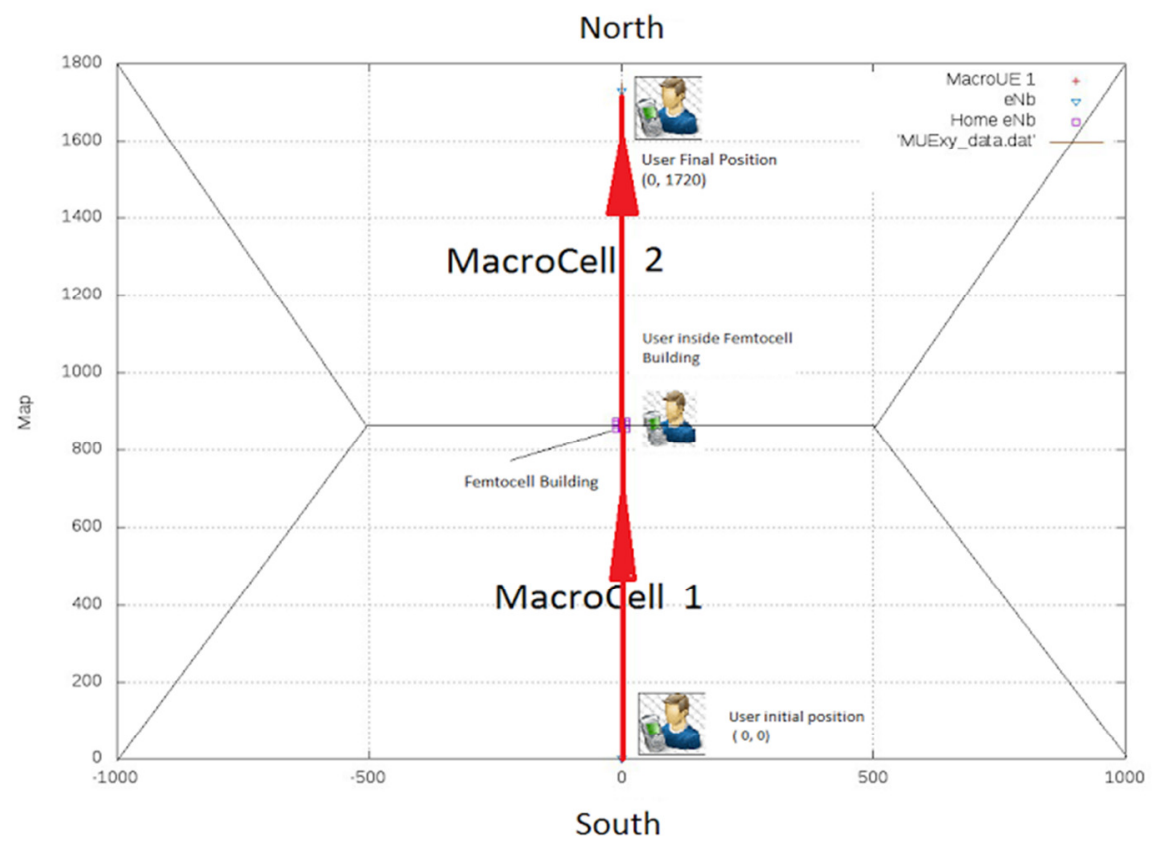

Fig.8: Mobility Pattern of the UE 


\section{Simulation RESUlT}

In this section, some simulation outcomes regarding the change in SINR of UE in different coverage area with and without presence of Femtocells are shown. The simulation results show: (i)SINR improvement of the UE as it moves from macro-cellular coverage to femto-cellular coverage area when FFR was not implemented, (ii) the SINR of the user further improves when FFR was implemented (iii) a scalability test on computational requirements (i.e., simulation time and CPU usage) was also performed at the end. Major simulation parameters are summarized in Tab. 1. In our simulation, presences of two eNBs were considered along with the UE having the access permission to all the femtocells. Building containing the femtocells in a $3 \times 3$ pattern is located at the cell edge of two cells.

Table 1: Simulation Parameters

\begin{tabular}{|l|l|}
\hline Total Bandwidth & $5 \mathrm{MHz}$ \\
\hline eNb power transmission & $43 \mathrm{dBm}$ \\
\hline FAP power transmission & $20 \mathrm{dBm}$ \\
\hline CQI & Full Bandwidth and periodic (2ms) reporting scheme \\
\hline Apartment Size & $100 \mathrm{~m}^{2}$ \\
\hline Mobility Model & Random Direction \\
\hline HO Type & Position Based HO \\
\hline Femto cell/Apartment & $1 / 1$ \\
\hline Frequency Reuse & Soft FFR, Strict FFR, FFR3 \\
\hline Apartment orientation & $3 \times 3$ \\
\hline Buildings & 1 \\
\hline Users & 1 \\
\hline Scheduler & PF \\
\hline Traffic & VOIP, VIDEO \\
\hline
\end{tabular}

As we can see from the simulation result, illustrated in Fig. 9 that, when the UE is moving from its initial position of Macrocell 1 coverage area to cell edge both the uplink and downlink SINR is decreasing because of path-loss, penetration and macro-macro co-channel interference. Position based HO model is considered in this simulation. As the UE moves closer to the FAP, HO takes place from eNB to FAP and it can be seen from Fig. 9 that the SINR has rapid improvement inside the FAP coverage area. Since we considered $3 \times 3$ apartment grid, it is also seen from the figure that, UE passed three FAP coverage area and 3 successful $\mathrm{HO}$ attempts were made. The fluctuation (three peaks in SINR graph inside FAP area) in SINR seen if Fig. 9 is because of the handovers to three different femtocells. As the UE passed the FAP coverage area, SINR has upward trend since, UE is moving towards the cell centre area of the Macrocell 2 coverage area. 
International Journal of Wireless \& Mobile Networks (IJWMN) Vol. 6, No. 6, December 2014

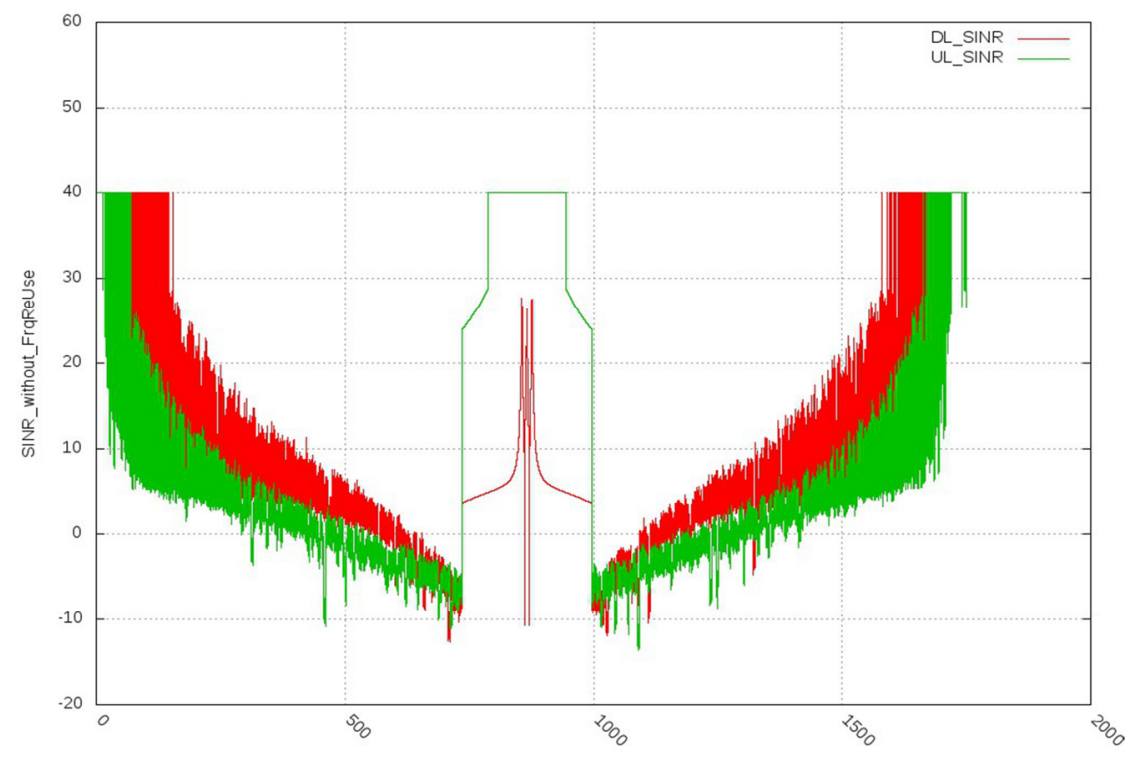

Fig.9: SINR without Frequency Reuse

When FFR is implemented DL SINR improves, which can be seen in Fig.10. FFR has no effect on uplink SINR since single UE is considered with no interference just path -loss, penetrationloss, multipath and shadowing.

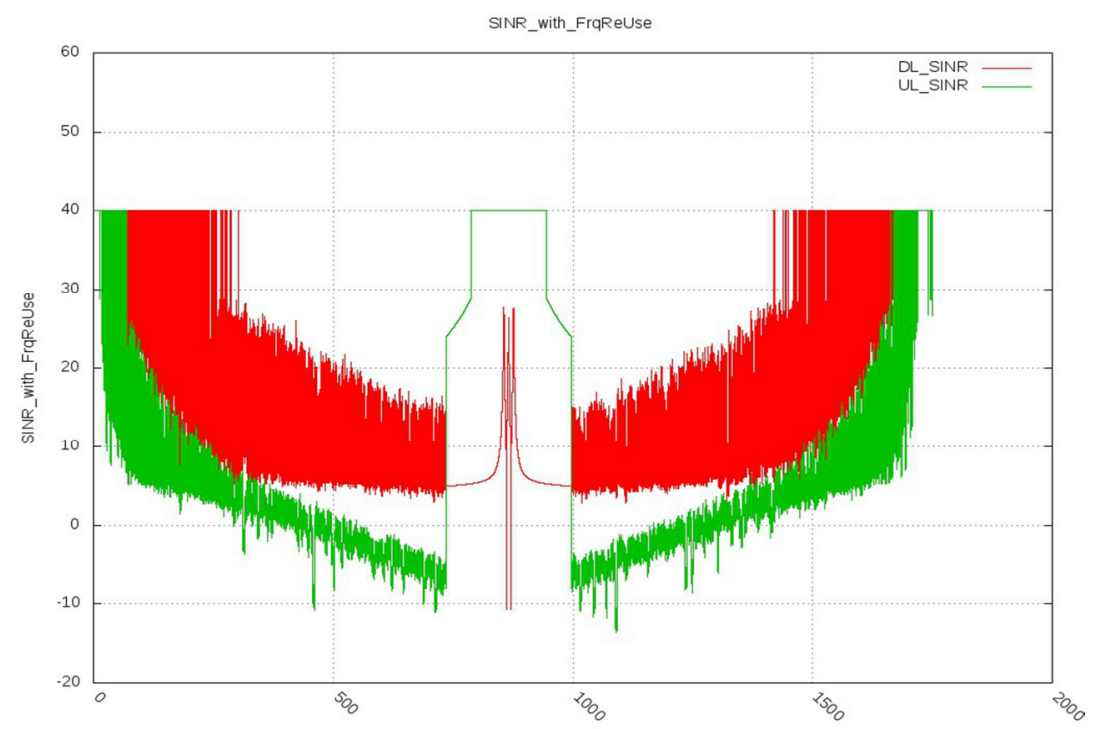

Fig. 10: SINR with Frequency Reuse

For more performance evaluation in different frequency reuse schemes i.e., FFR-3, Soft Frequency Reuse and Strict Frequency Reuse in with presence of increasing number of femtocells (maximum of 40 femtocells) are installed randomly inside the of the macrocell coverage. Fig. 11 shows the outage probability in different reuse schemes in different threshold SINR. Strict FFR 
International Journal of Wireless \& Mobile Networks (IJWMN) Vol. 6, No. 6, December 2014

has better performance than the other two this is because in Strict FFR the cells centre FUEs are not interfered by the FUEs of the edge area.

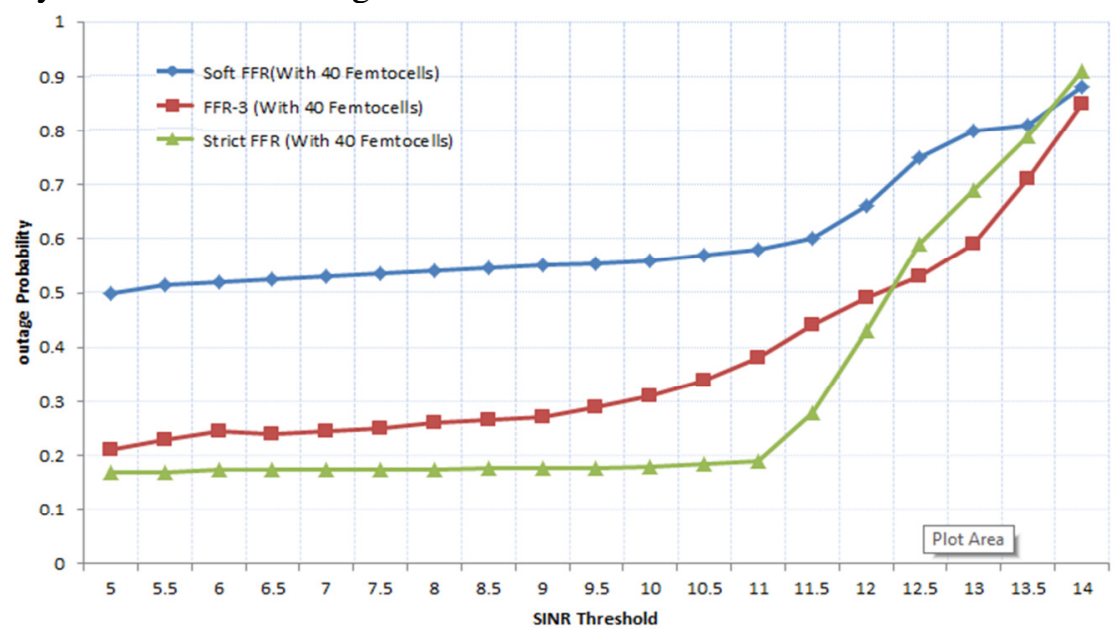

Fig. 11: Outage Probability Vs SINR Threshold in presence of Femtocells

The change in network throughput with the increase in number of femto cells is shown in Fig.12. With the increase in femtocell number throughout the macrocell coverage the network throughput decreases because of co-tier and cross tier interferences. FFR-3 schemes show the better network throughput.

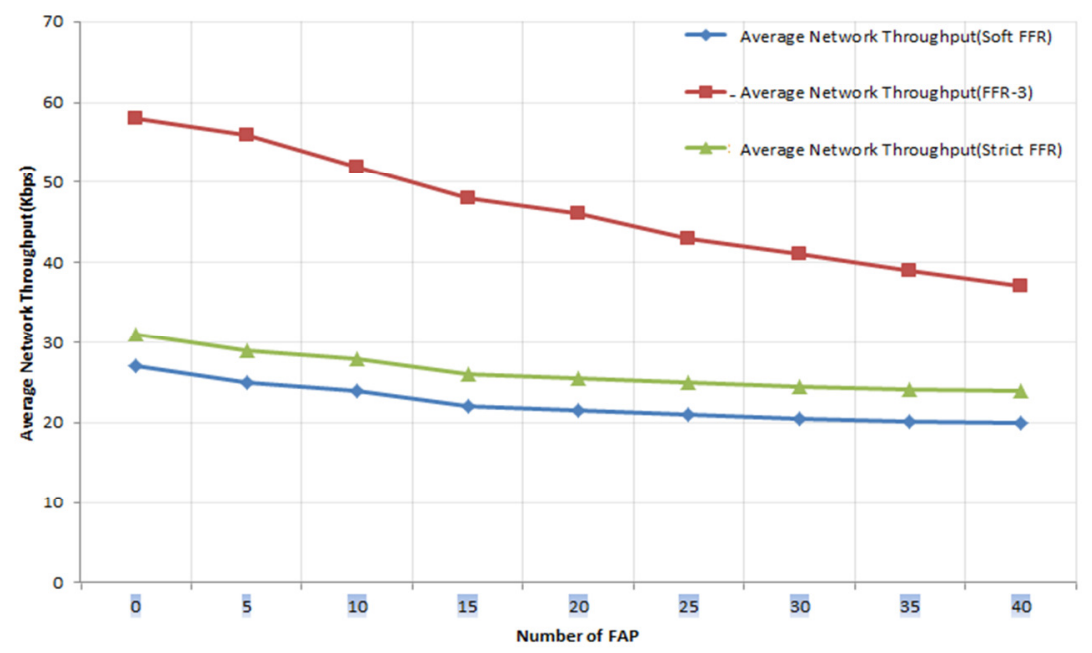

Fig. 12: Variation of Network Throughput with femtocell numbers

Fig.13 shows the spectrum efficiency of different reuse schemes in both cell centre(along with edge) and edge. FFR-3 has the better spectral efficieny in both the case. 
International Journal of Wireless \& Mobile Networks (IJWMN) Vol. 6, No. 6, December 2014

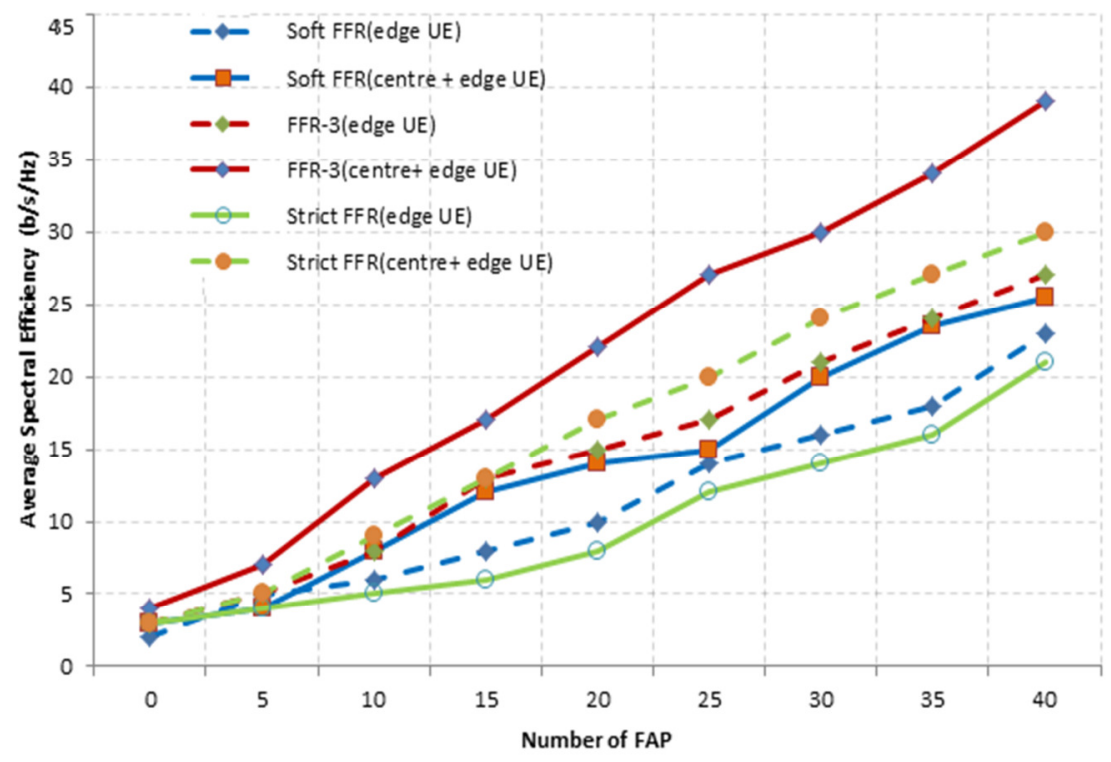

Fig. 13: Spectral Efficiency of different frequency Reuse schemes

The scalability test of the presented simulation model was running on Linux machine with a 3.2 $\mathrm{GHz}$ (Dual Core) CPU and 8Gbytes of RAM. Obtained measurements in Fig. 14 shows the simulation time and memory usage linearly increases with the increasing number of users and it can be seen in Fig. 14c that the CPU usage (1 out of 4 cores was fully used) remains the same in the scenarios with the highest computational load, which proofs the scalability of the presented model in LTE-Sim.
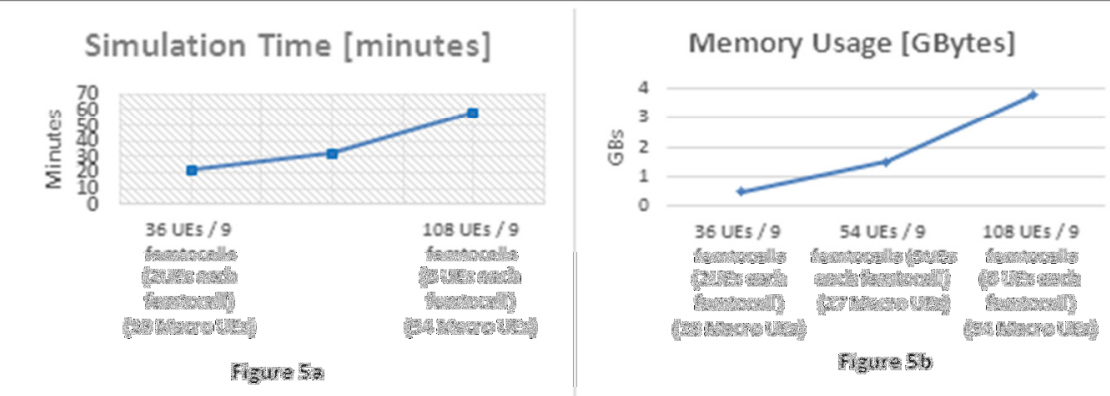

cou Usage Dpercentage W I

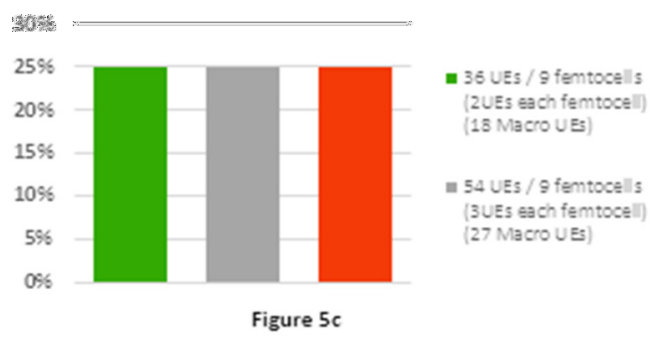

Figure 14: Simulator Performance in terms of a) Simulation Time b) Memory Usage c) CPU Usage(percentage \%) 
International Journal of Wireless \& Mobile Networks (IJWMN) Vol. 6, No. 6, December 2014

\section{CONCLUSION}

In this article, demonstration to simulate femtocell scenarios with features and models compliant with 3GPP specifications in LTE-Sim framework is provided. The femtocell scenario shown in this paper can be used for investigating performances in a wider range that can differ in terms of network layouts (i.e., number and position of base stations, building, and streets), system load (i.e., number of users and applications) and so on. As a future study, we plan to enhance the LTESim Femtocell framework with the aim of implementing mobility management, Handover, Radio Resource Allocation schemes [13] with random users both in eNB and FAP, handover, radio resource allocation schemes [21] as well as other algorithms and protocols based on both selforganizing network (SON) and cognitive paradigm believing the extreme modularity of LTE-Sim.

\section{REFERENCES}

[1] L. Nuaymi, WiMAX: Technology for Broadband Wireless Access. Wiley, NewYork, 2008.

[2] E Dahlman, S Parkvall, J Skold, P Beming, 3G Evolution HSPA and LTE for Mobile Broadband. Academia Press, USA, 2008.

[3] F. Capozzi, G. Piro, L. Grieco, G. Boggia, P. Camarda, "On Accurate simulations of LTE femtocells using an open source simulator," in EURASIP Journal on Wireless Communication and Networking, 2012.

[4] G Piro, L Grieco, G Boggia, F Capozzi, P Camarda,'Simulating LTE cellular systems: an opensource framework, " in Vehicular Technology, IEEE Trans. vol. 60, pp. 498-513, 2011.

[5] Raheem, Rand, Aboubaker Lasebae, and Jonathan Loo. "Performance Evaluation of LTE Network via Using Fixed/Mobile Femtocells." Advanced Information Networking and Applications Workshops (WAINA), 2014 28th International Conference on. IEEE, 2014.

[6] WCDMA for UMTS - HSPA Evolution and LTE, Fourth Edition. Edited by Harri Holma and Antti Toskala (C) 2007 John Wiley \& Sons, Ltd. ISBN: 978-0-470-31933-8

[7] D. Lopez-Perez, I. Guvenc, G. de la Roche, M. Kountouris, T. Q. S. Quek, and Z. Jie, "Enhanced intercell interference coordination challenges in heterogeneous networks," IEEE Wireless Commun., vol. 18, pp. 22-30, 2011.

[8] D. Xenakis, N. Passas, L. Merakos, C. Verikoukis, "Mobility Management for Femtocells in LTEAdvanced: Key Aspects and Survey of Handover Decision Algorithms" in IEEE Trans. Wireless Commun., vol. 18 pp. 2011.

[9] R. Bendlin, V. Chandrasekhar, C. Runhua, A. Ekpenyong, and E. Onggosanusi, "From homogeneous to heterogeneous networks: A 3GPP Long Term Evolution rel. 8/9 case study," in 45th Annu. Conf., Inform. Sci. and Syst. (CISS), Baltimore, MD, pp. 1-5, 2011.

[10] H. Mahmoud, I .Guvenc," A comparative study of different deployment modes for femtocell networks," in IEEE Int. Symposium on Personal, Indoor and Mobile Radio Commun. , Palo Alto, USA, pp. 1-5, 2009.

[11] Siemens, R1-060135, "Interference Mitigation by Partial Frequency Reuse," 3GPP RAN WG1\#42, London, UK, January 2006.

[12] T. Camp, J. Boleng, and V. Davies, "A survey of mobility models for ad hoc network research," Wireless Communications and Mobile Computing, vol. 2, pp. 483-502, Aug. 2002.

[13] F. Capozzi, G. Piro, L. Grieco, G. Boggia, P. Camarda, " A system-level simulation framework for LTE Femtocell," in Proc. of International Conference on Simulation Tools and Techniques(ICST), Desenzano, Italy, pp.1-3, 2012.

[14] http://www.infoutils.com/frequency-reuse-in-ofdma-based-ltewimax-system/ 
International Journal of Wireless \& Mobile Networks (IJWMN) Vol. 6, No. 6, December 2014

\section{Authors}

Shiqi Xing is currently doing his Bachelor of Telecommunication Engineering at University of Technology, Sydney. He is an experienced programmer and currently undertaking projects in $4 \mathrm{G}$ Telecommunication and Robotics. Throughout his academic career he received several scholarships from The People's Republic of China.

Pantha Ghosal is a Graduate Research Assistant at Faculty of Engineering and Technology (FEIT), CRIN, University of Technology, Sydney. Prior to this, he completed B.Sc in Electrical and Electronic Engineering from Rajshahi University of Engineering \& Technology, Bangladesh in 2007. He is an expert of Telecommunication network design and holds more than 7 years of working experience in $2 \mathrm{G} / 3 \mathrm{G}$ and LTE. Throughout his career he was involved in several projects of RF Planning, Designing and Dimensioning.

Shouman Barua is a PhD research scholar at the University of Technology, Sydney. He received his BSc in Electrical and Electronic Engineering from Chittagong University of Engineering and Technology, Bangladesh and $\mathrm{MSc}$ in Information and Communication Engineering from Technische Universität Darmstadt (Technical University of Darmstadt), Germany in 2006 and 2014 respectively. He holds also more than five years extensive working experience in telecommunication sector in various roles including network planning and operation.

Ramprasad Subramanian is an experienced telecom engineer in the field of 2G/3G and LTE/LTE-A. He holds M.S (By research) in Information and Communication from Institute of Remote Sensing, Anna University (India)(2007) and Bachelors of Engineering in Electronics and Communication engineering from Bharathidasan University (2001)(India). He has done many projects in the area of 2G/3G and LTE. He has done many consultative projects across Africs/Americas/Asia etc. He was the recipient of India's best invention award for the year 2004 from Indian Institute of Management Ahmadabad and Government of India. His current research focuses on 4G mobile networks and vehicular Ad hoc networks.

Dr Kumbesan Sandrasegaran is an Associate Professor at UTS and Centre for Real-Time Information Networks (CRIN). He holds a PhD in Electrical Engineering from McGill University (Canada)(1994), a Master of Science Degree in Telecommunication Engineering from Essex University (1988) and a Bachelor of Science (Honours) Degree in Electrical Engineering (First Class) (1985). His current research work focuses on two main areas (a) radio resource management in mobile networks, (b) engineering of remote monitoring systems for novel applications with industry through the use of embedded systems, sensors and communications systems. He has published over 100 refereed publications and 20 consultancy reports spanning telecommunication and computing systems. 